First publ. in: Physica / B + C, Vol. 126 (1984), 1-3, pp. $92-99$

\title{
ELECTRONS AT HELIUM INTERFACES
}

\author{
P. LEIDERER \\ Fachbereich Physik, Johannes Gutenberg-Universität, D-6500 Mainz, FRG *
}

Two-dimensional layers of charges trapped at the boundaries between the various helium phases strongly interact with these interfaces at high electric fields. The coupling, which leads to an electrohydrodynamic instability, provides new methods for studying helium properties.

\section{INTRODUCTION}

Electrons at the surface of liquid helium provide a somewhat exotic, yet in many respects ideal, model system for both theorists and experimentalists. Most thoroughly investigated are electrons above liquid helium, which in the direction perpendicular to the surface are trapped in the potential well arising from the image charges in the helium substrate, but along the surface can move as free particles - an example of an extremely clean two-dimensional coulomb system. Among the numerous experiments on this system are studies of single particle properties, such as the electronic effective mass and mobility, but also of collective phenomena like two-dimensional plasmons. Recently particular attention has been paid to the transition from an electron gas to an electron solid, which occurs at high charge densities and low temperature, where the coulormb interaction outweighs the thermal energy of the electrons.

All these results have been described in detail in a number of review articles (1). This paper will concentrate on some effects arising from the interaction between the layer of charges and the liquid substrate. In addition to electrons at the free liquid surface (i.e., the liquid-vapor interface) also liquid-liquid and liquid-solid interfaces of helium will be considered, and also positive charges at these interfaces will be discussed briefly.

\section{TRAPPING OF CHARGES AT HELIUM INTERFACES}

\subsection{Negative charges.}

If one wants to accumulate a charged $2 \mathrm{D}$ layer, the trapping time of the charges at the respective interface must be long. For electrons at the liquid-vapor interface this condition is fulfilled: Since the energy of a bare electron in liquid helium is about $1 \mathrm{eV}$ higher than in the gas (1), electrons pulled from the vapor side onto the liquid by a suitable electric field encounter an energy barrier $\Delta W$ huge compared to the thermal energy at $1 . K$. They therefore cannot enter the liquid - provided they have not picked up too much energy in the applied field - and can thus be stored practically indefinitely, forming a sheet which floats about $100 \AA$ above the surface.

In order to charge the other interfaces which are considered here electrons in liquid or solid helium are required, and there the situation becomes somewhat more subtle. An electron, injected into one of the condensed phases with sufficiently high energy, relaxes rapidly into a new state where it resides in a bubble (some $40 \AA$ in diameter at svp), thereby decreasing its energy from $1 \mathrm{eV}$ to $0.2 \mathrm{eV}$ (2). Also for these electron bubbles - or "negative ions" - an energy barrier $\Delta W$ may exist at the boundary between two different phases. An order of magnitude estimate for $\Delta W$ can be obtained

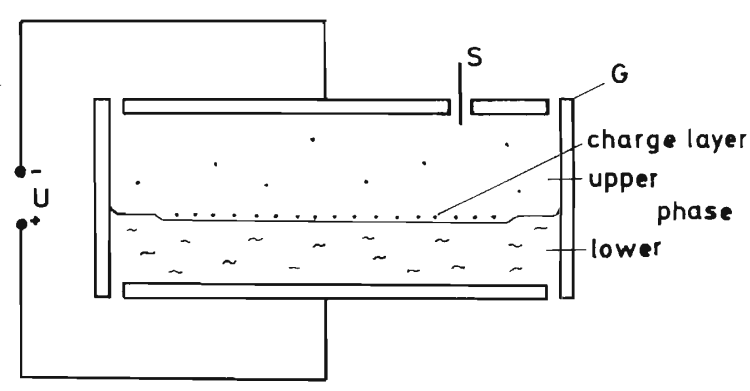

FIGURE 1

Schematic experimental set-up used to study charge carriers at helium interfaces. The bottom and top capacitor plates supply the electric holding field; the guard ring $G$ prevents the charges from leaking to the walls. The source $S$ for the charge carriers my be a field emission tip, corona discharge, heated filament, tunnel cathode, or radioactive source. For optical measurements of the interfacial deformations the capacitor consists of glass plates with a transparent conductive coating.

* Work supported by the Deutscine Forschungsgerie inschaft 
from the total energy $W$ of a negative ion in the various phases, which is essentially given by

$$
\begin{gathered}
W=\frac{\hbar^{2} \pi^{2}}{2 m_{e} R^{2}}\left[1+\frac{\hbar}{R\left(2 m_{e} V\right)^{1 / 2}}\right]^{-2}+4 \pi R^{2} \sigma+\frac{4}{3} \pi R^{3} p \\
-e^{2}(\varepsilon-1) / 2 R \varepsilon
\end{gathered}
$$

Here the first rhs term represents the ground state energy of an electron with mass $m_{e}$ in a potential well of width $2 \mathrm{R}$ and depth $\mathrm{V} \sim 1 \mathrm{eV}$ (given by the above mentioned energy difference between an electron in vacuum and bulk helium), the second term results from the surface energy of the bubble ( $\sigma$ is the surface tension of helium), the third from the hydrostatic pressure $p$, and the last one from the polarization of the bulk phase with a dielectric constant $\varepsilon$. Neglected are here corrections such as a possible dependence of the surface tension on the radius of curvature or anisotropy effects in the crystalline phase. From a minimization of the resulting bubble energy with respect to $R$ the equilibrium radius and the energy difference of the bubbles, $\Delta W$, between the respective coexisting phases can be determined (3). Examples are:

- for the liquid-solid ${ }^{4}$ He system the bubble energy is expected to be higher in the crystalline phase by an amount $\Delta W=18 \mathrm{meV}$ at a temperature $\mathrm{T}=1 \mathrm{~K}(4)$

- for bubbles in a phase separated ${ }^{3} \mathrm{He}-{ }^{4} \mathrm{He}$ mixture, a liquid-liquid system, the energy in the lower, $4 \mathrm{He}$ rich phase at $0.3 \mathrm{~K}$ is estimated to be 20 meV higher than in the upper phase (5).

The reason why in both cases the energy in the lighter phase is reduced is related to the smaller surface tension of the lower density phases.

With the energy differences $\Delta W$ being large compared to the thermal energy, as before, one expects that also $2 \mathrm{D}$ layers of electrons in bubbles can be accumulated at the interface of $3 \mathrm{He}-4 \mathrm{He}$ mixtures and at the liquid-solid boundary of $4 \mathrm{He}$. This has been confirmed experimentally by studying the trapping of negative ions with an optical technique, where the slight depression of the interface (6) due to the ion pressure was recorded as a function of time. No change of the ion density was observed on a time scale of hours, indicating that indeed both negative ions above the $3 \mathrm{He}-4 \mathrm{He}$ interface and above the crystal surface of 4 He can be trapped over long periods $(3,4)$.

\subsection{Positive charges.}

Positive ions in liquid He are supposed to consist of a "snowball" of solid $\sim 15 \AA$ in diameter around the central positive charge, which may be a $\left(\mathrm{He}_{2}\right)+(2)$. The energy of such ions then is made up of the energy of the solid core (which probably does not change as an ion passes through an interface) and the energy contributions from the interfacial tension between solid and liquid and from the polarization of the surrounding medium. Since the latter terms both favor the high-density bottom phase, interfacial energy barriers ought to exist in just the opposite direction compared to negative ions - namely from the lower to the upper phase. It therefore should be possible to store positive ions just underneath the various helium interfaces by applying a suitable electric field which pulls them towards the boundary from below. For the liquid-gas and liquid-liquid interface this prediction has been verified $(1,3)$.

Another energy contribution not mentioned so far arises from the image potential of the charges in the vicinity of an interface. Due to the resulting local peak in the potential also negative ions encounter an energy barrier - although relatively small - when pulled against, say, the liquid vapor interface from below. Therefore, in addition to the examples quoted here more combinations of 2 D charge systems and He interfaces can be prepared and have, in fact, been studied successfully (1).

3. INTERACTION BETWEEN THE ELECTRONS AND INTERFACIAL EXCITATIONS: THE EHD INSTABILITY

3.1. Softening of interfacial waves

The excitation spectrum of waves at an (uncharged) liquid-gas or a liquid-liquid interface is given by

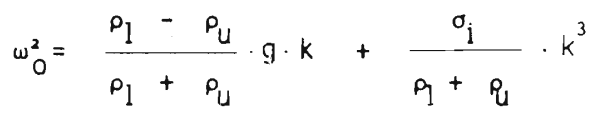

where it is assumed that the depth of the liquid is large compared to $1 / k$, and that dissipation is negligible. Here $\omega_{0}$ and $k$ are the frequency and wave vector of the excitation, $g$ is the acceleration due to gravity, $\rho_{u}$ and $\rho_{l}$ are the densities of the upper and lower phase, and $\sigma_{i}$ is the interfacial tension.

When electrons are put onto the interface, interfacial excitations interact with them via electron-ripplon scattering, a process which, for example, at low temperatures limits the mobility of the free electrons above the liquid surface (1). Vice versa, the electron layer also affects the excitation spectrum of the rioplons by reducing the restoring forces, which gives rise to an additional (negative) term in the dispersion relation $(7,8)$ :

$$
\omega^{2}=\omega_{0}^{2}-\frac{\left(E_{1}^{2}+E_{u}^{2}\right)}{4 \pi\left(\rho_{1}+\rho_{u}\right)} \cdot k^{2}
$$

Here $E_{1}$ and $E_{u}$ are the static fields below and above the interface, and we have assumed that the charge carrier mobility is high enough so that the interface is always an equipotential. 
The effect of the charges at the interface expected according to $\mathrm{Eq} .3$ is sketched in Fig. 2 . The reduction of the ripplon frequency is most pronounced at wave vectors around the inverse capillary length $1 / \mathrm{a}=\left[\left(\rho_{1}-\rho_{\mathrm{u}}\right) \mathrm{g} / \sigma_{\mathrm{i}}\right]^{1 / 2}$, the transition range from gravitational to capillary interfacial waves. The data for a charged $3 \mathrm{He}-{ }^{4} \mathrm{He}$ interface (9) also plotted in Fig. 2 show that the agreement with the predicted behavior is quite satisfactory.

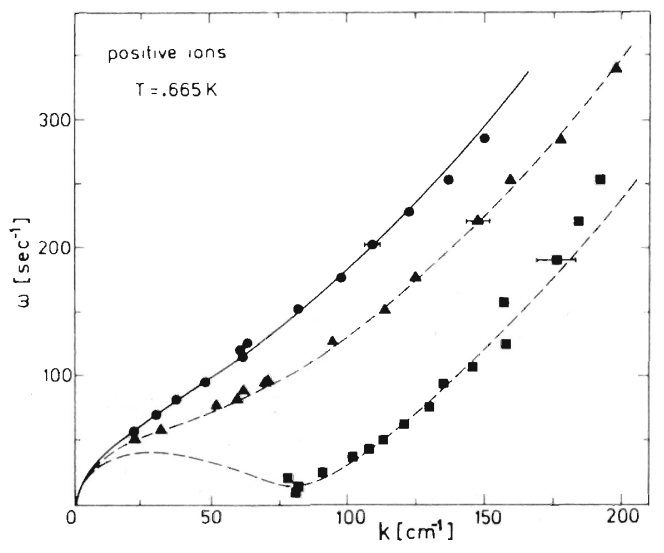

FIGURE 2

Dispersion relation of ripplons at the interface of a phase separated $3 \mathrm{He}-{ }^{4} \mathrm{He}$ mixture completely charged with positive ions. The interface becomes unstable at $E_{C}=875 \mathrm{~V} / \mathrm{cm}$, corresponding to an ion density $\mathrm{n}_{\mathrm{C}}=4.8 \times 10^{8} \mathrm{~cm}^{-2}$. $\bullet, E / E_{C}=0.12$; $\Delta, 0.71 ; 0,0.995$. The dashed curves are calculated according to $\mathrm{Eq}$. (3). The dispersion of the uncharged interface is given by the solid line (Ref. 10).

The softening of interfacial waves is reminiscent of structural phase transitions, where a soft mode signals that a system approaches some instability point. In fact, also for charged helium interfaces an instability and a transition to a state with a new symmetry is observed, as discussed in the following paragraph.

3.2. The formation of the dimple lattice For an interface charged to saturation the electric field in one of the phases is screened completely by the interfacial electron or ion density $n$, while in the other it is $E=4 \pi n$. As seen from Eq. 3 and $\mathrm{Fig} .2$, the frequency of the soft ripplon mode at $k=1 / a=k_{c}$ goes to zero when the charge density reaches a critical value $n_{c} e=\left[\left(\rho_{1}-\rho_{u}\right) g \sigma_{i} / 4 \pi^{2}\right]^{1 / 4}$ at a corresponding critical electric field $E_{C}$. For $n$ just above $n_{c}$ w becomes imaginary at a wave vector $k=k_{c}$, which implies that fluctuations in the elongation of the interface with that particular k-value will increase in time instead of decay. As a result, a disruptive loss of charge from the interface develops (11).

A qualitatively quite different feature of the electrohydrodynamic (EHD) instability is observed, when the surface is not charged to saturation, but only to about 10 percent of $n_{C}$ or less (12). Although again interfacial waves with $k=k_{c}$ become unstable as the electric field is raised somewhat above a critical value $E_{C}$, and consequently the interface ripples spontaneously with a characteristic wavelength $\lambda_{C}=2 \pi / k_{C}$, nonlinear contributions to the restoring force restrict these deformations to a finite amplitude. Thus a new equilibrium state in the charge distribution develops, different from the homogeneous layer for fields below $E_{C}$.

Fig. 3 shows the symmetry of the charge distribution in this state for the example of electrons at the 4 He free liquid surface. In this photograph, taken by a Schlieren technique, local minima of the deformed surface - which coincide with maxima in the electron density appear bright. Obviously the electrons aggregate in clusters, leading to dimples at the surface, which form a regular lattice. Since this triangular "dimple crystal" has developed from the soft ripplon mode, its lattice spacing is close to $\lambda_{C}$.

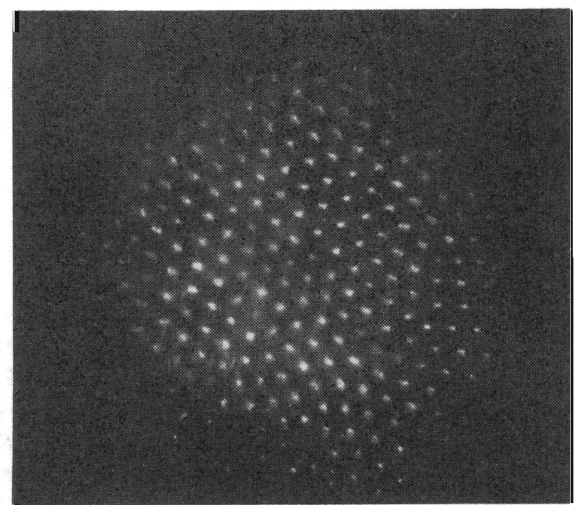

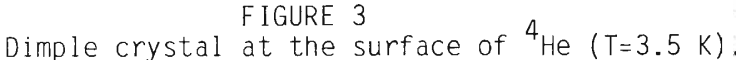
The distance $D$ between adjacent dimple rows is equal to the wavelength of the soft ripplon, $2 \pi / k_{C}, 0.24 \mathrm{~cm}$ in this case $(13)$.

The transition to the dimple state is reversible: By reducing the electric field the homogeneous charge distribution and the flat interface are retained. If the charge density is lower than the above mentioned limit of $\sim 0.1 \mathrm{n}_{c}$, the system can repetitively be cycled through the instability without losing charge. An analysis of this transition shows that - in 
terms of the language of phase transitions - it is of weakly first order $(14,15)$. Consequent ly hysteresis effects in the dimple formation are expected on varying the electric field $E$, which here plays the role of an effective temperature. such effects have been observed indeed in a range $\left(1-E / E_{C}\right)$ of the order of $10-2(12,16)$. Furthermore, in an experiment where the liquid surface was carefully shielded from external vibrations, a stripe phase has been found to exist in a certain range close to $E_{C}$. There the parallel corrugations, which develop from the soft ripplon mode, are stable and do not break up into the usual triangular array of dimples (16).

Another aspect of the dimple lattice follows from a consideration of the charge distribution in this state: As numerical calculations show $(13,17)$, the charge density is not just slightly. modulated along the surface, but is large in a region close to the dimple centres and zero elsewhere. Since the potential barrier between neighboring dimples is high, the amount of charge in each dimple is fixed, and as a result the interaction between the dimples is dominated by coulomb repulsion with only a small contribution from the deformation of the interface. The dimple lattice can therefore be regarded as an example of a 2 D Coulomb crystal. In fact, lattices like the one in Fig. 3 not only display the triangular symmetry expected for such a system, but also typical defects like dislocations and grain boundaries $(9,16)$. The dynamical properties of this crystal have not been investigated so far; it is to be expected, though, that because of the strong coupling to the interfacial modes of the liquid the excitations will differ considerably from those of a bare coulomb lattice.

The existence of multielectron dimples on liquid surfaces does not depend on the formation of a more or less regular array. If only few electrons are present at the surface and the electric field is chosen properly, dimples can develop and are stable also as single, isolated objects. Characteristic properties like the width, depth, and eigenfrequencies of such individual dimples have been determined by several authors $(13,15,17,18,30)$. Typically, a dimple containing $5 \times 10^{6}$ electrons is a few tenths of a mm deep and has a width of the order of the capillary length, which is in the $\mathrm{mm}$ range.

\subsection{The growth of the EHD instability}

For electric fields distinctly higher than $E_{C}$ the restoring force due to the deformation is no longer sufficient to hold the charge at the interface. The resulting break-through can be described, in its initial stage, by an extension of Eq. 3, which now in a certain band of wave vectors yields unstable solutions. The range of unstable modes is plotted in the stabil-. ity diagram in Fig. 4 as a function of the reduced electric field $E / E_{C}$ :

- for $E<E_{C}$ the system is stable for all values of $k$

- at the instability threshold $E=E_{C}$ the mode with $k=k_{c}$ becomes unstable

- for $E>E_{C}$ the unstable range, where $a$ perturbation grows instead of decays, covers an increasing band of wave vectors.

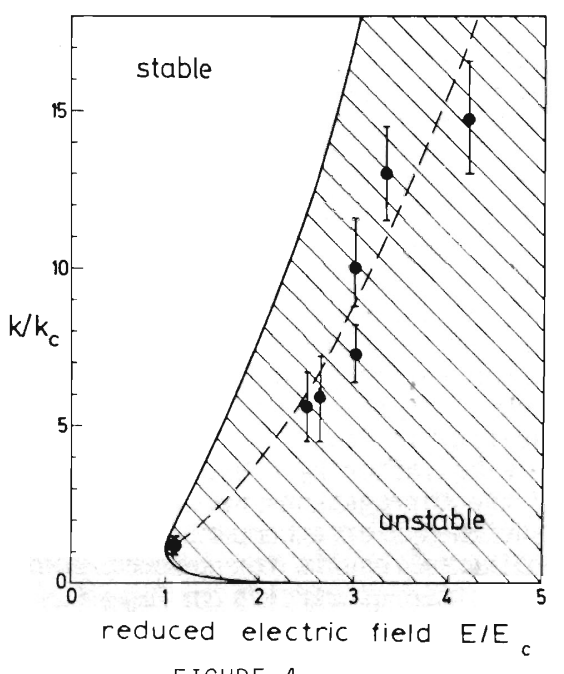

FIGURE 4

Stability diagram of a charged interface, as calculated from Eq. 3. The dashed line indicates the unstable wave vectors with the largest gain. The data points represent experimental results of $\mathrm{k}_{\mathrm{m}}$ (see below) for the hcp-superfluid $\mathrm{He}$ interface (4).

In general, the "punch-through" process of the charges leads to a severe perturbation of the interface. The electrohydrodynamic deformations grow in amplitude, until charged bubbles split off and move to the counter electrode. For the liquid-gas interface this process, which takes place within milliseconds, has been recorded with a high speed camera (11). Since the damping of surface waves in this case is small, the perturbations add up and the whole interface immediately is in chaotic motion. An interface which allows to observe the development of the EHD instability under better - controlled conditions and in somewhat more detail is provided by the boundary between liquid and solid 4 He. As shown by Andreev and Parshin (19) and Keshishev et al. (20), at this interface propagating melting - crystallization waves exist whose excitation spectrum resembles that of ripplons at a liquid surface (Eq. 2). The attenuation of these waves depends strongly on 
temperature; above $1 \mathrm{k}$ they are overdamped in the critical wave vector range around the inverse capillary length (4). When such an interface is charged with ions, the stability diagram shown in Fig. 4 should also apply. As a technical advantage, in this case the perturbing influence of waves reflected from the container walls, which usually obscure the growth of the EHD instability, is suppressed because of the strong damping, and the growth of the unstable modes can easily be followed on a time scale of seconds. A picture of such an interface "quenched" into the unstable regime by a sudden increase of the electric field is presented in Fig. 5. Although the pattern is not nearly as regular as the dimple lattice in Fig. 3, some periodicity in the deformation of the crystal surface can still be recognized. Apparently among the wide band of competing unstable modes at that field a certain, relatively small wave vector range dominates the growth. The orientation of the corrugations seen in Fig. 5 is related to the anisotropy of the ${ }^{4} \mathrm{He}$ crystal.

The higher the applied field the faster this instability is found to develop. Simultaneously the wave vector $k_{m}$ characterizing the spacing of the corrugations becomes larger and less well-defined. For a comparison with the stability diagram results for several electric fields are plotted as circles in Fig. 4.

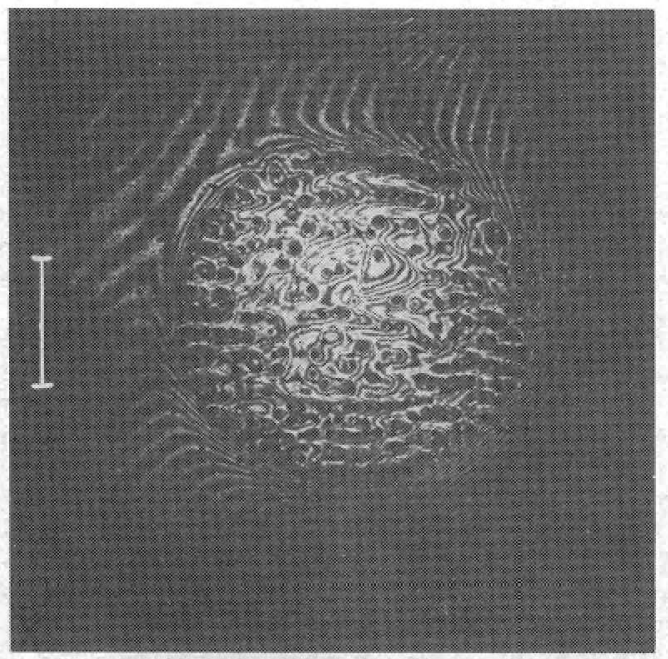

FIGURE 5

Snapshot of the instability pattern developing at an hcp-superfluid ${ }^{4} \mathrm{He}$ interface at $E / E_{C}=2.6$. The characteristic distance of the stripes is distinctly smaller than the lattice spacing of the dimple crystal at the critical field, $2 \pi / k_{c}=6.3 \mathrm{~mm}$ (indicated by the scale bar).
The development of this instability resembles closely, in certain respects, the phenomenon of spinodal decomposition in two-component liquids or alloys with a miscibility gap. When such a system is quenched from the one-phase state through the consolute critical point into the unstable range, it starts to phase-separate spontaneously. This process is characterized by the instability of a whole range of wave vectors, but as for the EHD instability a particular k-value grows most quickly and dominates the decomposition, resulting in patterns similar to Fig. 5. The charge-induced instability at helium interfaces thus can be considered in the context of a much wider class of critical phenomena

\section{SURFACE ELECTRONS AS A PROBE OF HELIUM PROPERTIES}

The phenomena described in the previous sections can not only be treated as problems of their own right, they also allow to gain information about the properties of the various helium interfaces and the structure of the charge carriers. Some examples for this application of surface charges will be given below.

\subsection{Trapping time of charges.}

The energy barriers $\Delta W$ at the interfaces thus far have been assumed to be so large compared to the thermal energy of the charges that the probability to pass the barrier was negligible, and the trapping time $\tau$ at the interface hence was arbitrarily long. This assumption certainly does not hold near a critical point where the two coexisting phases become identical and $\Delta W$ therefore has to vanish. A system which has been studied in this respect is the charged interface of ${ }^{3} \mathrm{He}-{ }^{4} \mathrm{He}$ mixtures in the vicinity of the tricritical point at $T_{+}=0.867 \mathrm{~K}$ (3). As $T_{t}$ is approached $\tau$ drops sharply by many orders of magnitude both for negative ions above and for positive ions below the interface. The data suggest a dependence $\tau \propto \exp \left(\Delta W_{+} / K T\right)$ with the energy barriers $\Delta W_{+}$ and $\Delta W_{-}$for the positive and negative ions, respectively, varying roughly as $\Delta W \propto\left(T_{t}-T\right)$. The experimental values for $\Delta W$ have been used to test the existing snowball and bubble models (Eq. 1). Although qualitative agreement is obtained, the details of the data are not reproduced, which calls for more defined theories for ions in $3 \mathrm{He}-{ }^{4} \mathrm{He}$ mixtures.

A related problem is the trapping of positive ions at the boundary between the liquid and the solid phase. Due to polarization effects the energy of the snowball ought to be lower in the solid, so that one might expect these ions to pass easily from the liquid side into the crystal. On the other hand, the structure of a snowball should differ significantly from that of the high quality single crystals formed by bulk solid He. This is supported by the obser- 
vation that positive ions do not grow in size even if the surrounding pressure is increased up to the solidification of the bulk phase (21): snowballs therefore do not act as nucleation centres for crystalline He - an indication that an additional interfacial energy between the disordered snowball and the bulk crystal exists. The snowballs then might be prevented from penetrating into the solid by an interfacial potential barrier. However, experiments which we have performed show that the trapping times of positive ions on solid He are very short; at $T=1.3 \mathrm{~K}$ we estimate an upper limit of several seconds. The potential barrier imposed by the boundary between disordered and ordered solid He, if it exists, therefore must be smaller than or at most of the order of several kelvin.

4.2 The electrostatic pressure at $E<E_{C}$. Because of the pressure which they exert on the helium interfaces, electrons and positive ions can be used as a delicate tool to deform the interfaces in a controllable manner. Two examples, where this technique has been applied, are measurements of the crystal growth of $4 \mathrm{He}$ and the parametric excitation of surface waves:

a) When negative ions are pressed against the surface of a helium crystal, the chemical potential in the solid is increased, whereas in the liquid it is unchanged. Consequently, a thin crystal layer melts, until the electrostatic pressure $P_{e l}$ is compensated by the chemical potential difference due to gravity. The pressures which are applied here are minute: A depression $0.1 \mathrm{~mm}$ deep corresponds to $\mathrm{P}_{\mathrm{el}}=$ $1.8 \times 10^{-7}$ bar. As soon as the ion pressure is released, the interface starts to relax back to its original position. This can be measured, for instance, by optical techniques like interferometry. It is found that for temperatures above the roughening transition of the solidliquid interface (22) the relaxation closely follows an exponential behavior, confirming that for atomically rough surfaces no activation energy is required for crystal growth, and that therefore the growth velocity of the crystal is proportional to the chemical potential difference between liquid and solid. The relaxation time $\tau_{R}$ for this process is related to the growth coefficient $\left(m_{4} K\right)$ of solid He by (24)

$$
\tau_{\mathrm{R}}^{-1}=\left(\mathrm{m}_{4} \mathrm{k}\right) \cdot \mathrm{g} \cdot\left(\rho_{\text {sol }}-\rho_{\text {liq }}\right) / \rho_{\text {sol }}
$$

Some data for the growth coefficient determined in that way are plotted in Fig. 6, together with results obtained from the damping of melting - crystallization waves (20) and the transmission of sound through the interface (25). The data are compatible with a dependence

$$
\left(m_{4} k\right)^{-1} \text { a } \exp \left(-\Delta_{r} / k_{B} T\right)
$$

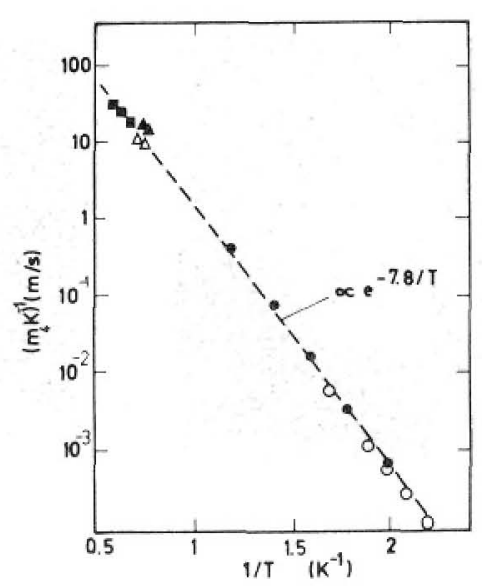

FIGURE 6
Inverse growth coefficient of solid ${ }^{4} \mathrm{He}$, bcc; $\Delta, \mathbf{\Delta}$, hcp; 0 , ref. (20); ref. (25). For the low temperature data the phonon contribution to the growth resistance has been subtracted.

where $\Delta r$ is the roton energy. The crystallization rate thus appears to be dominated by the thermal excitations in the liquid phase. The theories developed for the growth coefficient $(26,27)$ in their original form only account for the temperature dependence in the ballistic regime at low $T$, where the mean free path of the excitations is large; arguments to extend the validity so that in the whole temperature range displayed in Fig. 6 the relation (5) should hold have been presented by castaing (28).

b) Waves on charged helium surfaces are easily excited when the pressure of the electrons is periodically varied by slightly modulating the external holding field. As in other nonlinear systems the driving amplitude seriously influences the generation of the surface modes. An example is given in Fig. 7, which shows resonance modes on charged liquid 4 He in a cylindrical sample cell, as seen from above. The frequency of the sinusoidal driving field was $\omega \mathrm{ac}=300 \mathrm{~s}^{-1}$, and the surface was il luminated

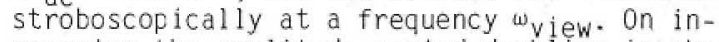
creasing the amplitude period doubling is observed, and finally a transition to chaotic motion occurs. The detailed "route to chaos" in this system, which is characterized by coupled ripplon-plasmon modes, is presently investigated (29).

4.3. Parameters of the dimple lattice The formation of the dimple lattice varies strongly near a critical point, where two rele- 

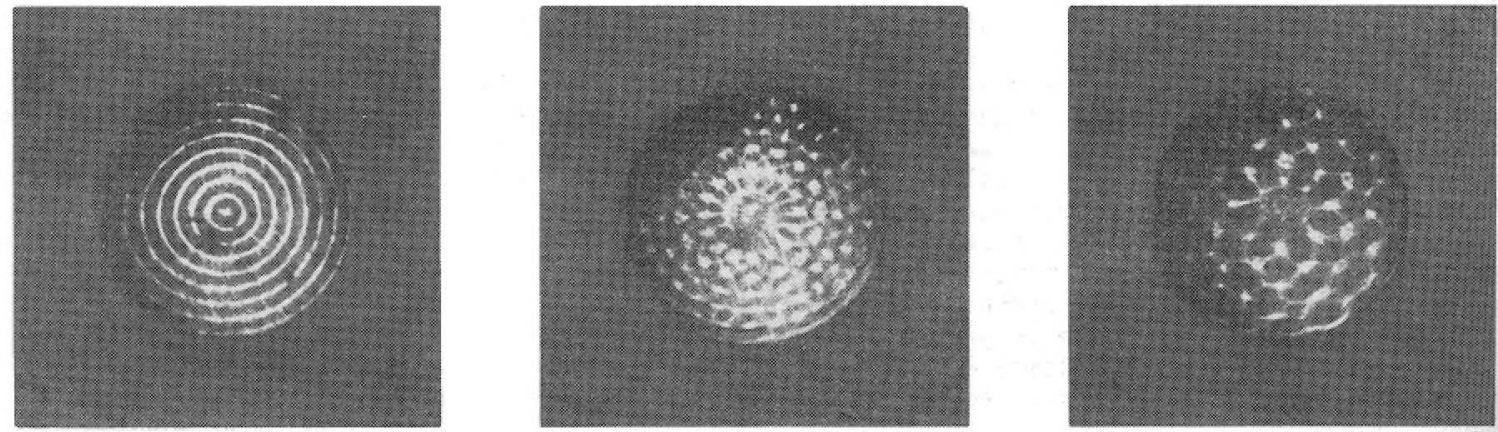

\section{FIGURE 7}

Surface modes on liquid "He charged with electrons. a) $\omega_{\text {view }}=\omega$, ac, low driving field; b) $\omega$ view ${ }^{4}$ ac' driving field increased; c) same field as in b), but at an illumination frequency $\omega_{v i e w}=\omega_{a c} / 2 . A$ comparison of the patterns b) and c) shows the effect of period doubling.

vant material parameters, $\sigma_{i}$ and $\Delta p$, vanish. This is illustrated in Fig. 8 for ${ }^{3} \mathrm{He}-{ }^{4} \mathrm{He}$ mixtures in the vicinity of the tricritical point at $T_{t}=0.867 \mathrm{~K}$.

The interfacial tension and the difference in the densities of the coexisting phases can be written in terms of $E_{C}$ and $k_{C}$ as

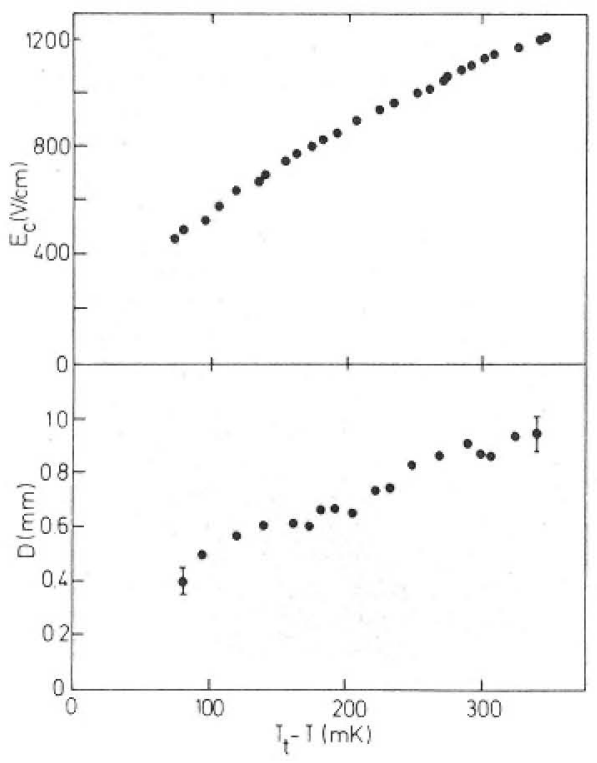

FIGURE 8

Critical field $E_{C}$ and spacing of the dimple lattice, $D=2 \pi / k_{C}$, in a phase separated ${ }^{3} \mathrm{He}-{ }^{4} \mathrm{He}$ mixture near the tricritical point.

$$
\begin{aligned}
\sigma_{i} & =E_{c}^{2} / 8 \pi k_{c} \\
\Delta p & =E_{C} 2_{k_{c}} / 8 \pi g
\end{aligned}
$$

Thus alone from a measurement of the parameters characterizing the dimple lattice the absolute values of $a_{i}$ and $\Delta \rho$ can be determined. Results obtained for $3 \mathrm{He}-{ }^{4} \mathrm{He}$ mixtures from $\mathrm{Fig} .8$ are in good accord with the known critical behavior of $\sigma_{i}$ and $\Delta \rho(10)$. Similarly, measurements at the liquid-solid and liquid-vapor interfaces $(24,12)$ have yielded interfacial tensions which confint the results of earlier experiments (23).

\subsection{Electrons on helium films}

The EHD instability limits the electron density on bulk liquid helium to $n<2.5 \times 10^{9} \mathrm{~cm}^{-2}$ Higher densities - which are of considerable interest in the context of the transition from a classical coulomb to a degenerate Fermi syste- are accessible on helium films supported by a solid substrate, because there the liquid surface is additionally stabilized by van der Waals forces. For a typical saturated film thickness $d=400$ \& the EHD instability is expected to occur at a critical electron density $n_{c}=$ $2 \times 10^{10} \mathrm{~cm}^{-2}$, the exact value depending somewhat on the substrate parameters $(1,31)$. An even higher density should be stable on thinner films, given by the relation (31)

$$
n_{C} \propto 1 / d
$$

because the van der Wals-interaction grows rapidly as d is decreased.

Experimental results on charged helium films are rather sparse, partly because the measurements are hamcered by a very low electronic 
mobility, which has been ascribed to the formation of one-electron dimples or "polarons" $(32,33)$. Experiments on the predicted ripplon softening as a precursor of the EHD instability have not yet been reported. What was found, however, were stable electron densities up to $10^{11} \mathrm{~cm}^{-2}$ on helium films above insulating substrates, and possibly somewhat less $\left(5 \times 10^{10} \mathrm{~cm}^{-2}\right)$ above metals (34-36). These values were nearly independent of the initial thickness of the uncharged film, in apparent contradiction to the relation (7). The reason is that in the existing theories $d$ has been treated as an independent variable, which can be chosen arbitrarily, whereas under usual experimental conditions the thickness of a saturated $\mathrm{film}$ is strongly related to the electron density and drops drastically on charging because of the electronic pressure. At high densities $d$ is mainly determined by the equilibrium between electric and van de kaals forces, with only a minor contribution from gravity. The thickness of these highly charged saturated films at $n \sim 10^{11} \mathrm{~cm}^{-2}$ is about 100 , as measured by ellipsometry (36). Which mechanism eventually limits the electron density - an EHD instability, or competing processes like tunneling of the electrons through the film - requires further study.

\section{ACKNOWLEDGEMENT}

Among the many collegues, who have contributed to the work reported here, I would I ike to thank in particular J. Bodensohn, W. Ebner, H. Etz, S. Herminghaus, V.B. Shikin, D. Savignac and M. Wanner.

REFERENCES

(1) See, e.g., M.W. Cole, Rev.Mod.Phys. 46(1974) 451; C.C. Grimes, Surf.Sci. 73 (1978) 379; V.S. Edelman, Sov.Physics-Uspekhi 23 (1980) 227; F.I.B. Will iams, Surf.Sci. 113 (1982) 371; Yu.P. Monarkha and V.B. Shikin, Sov.J. Low Temp.Phys. 8 (1982) 279

(2) A.L. Fetter, in: The physics of liquid and solid helium, eds. K.H. Bennemann and J.B. Ketterson (Wiley, New York, 1976) Vol. 1, p. 207

(3) P. Leiderer, M. Wanner, and W. Schoepe J.Phys. (Paris), Colloq. 39 (1979) C 6-1328

(4) D. Savignac and P. Leiderer, Phys.Rev. Lett. 49 (1982) 1869

(5) M. Kuchnir, P.R. Roach, and J.B. Ketterson, J.Low Temp.Phys. 3 (1970) 183

(6) R. Williams and R.S. Crandall, Phys.Lett. 36A. (1971) 35

(7) L.P. Gorkov and D.M. Chernikova, JETP Lett. 18 (1973) 68

(8) K. Mima and H. Ikezi, Phys.Rev. B 17 (1978) 3567

(9) M. Wanner and P. Leiderer, Phys.Rev.Lett. 42 (1979) 315; P. Leiderer, Phys.Rev. B 20 (1979) 4511

(10) P. Leiderer, H. Poisel, and M. Wanner, J. Low Temp.Phys. 28 (1977) 167
(11) A.P. Volodin, M.S. Khaikin, and V.S. Edelman, JETP Lett. 26 (1977) 543

(12) W. Ebner and P. Leiderer, Phys.Lett. $80 \mathrm{~A}$ (1980) 277

(13) P. Leiderer, W. Ebner, and V.B. Shikin, Surf.Sci. 113 (1982) 405

(14) H. Ikez1, Phys.Rev.Lett. 42 (1979) 1688

(15) V.B. Shikin and P. Leiderer, Sov.Phys. JETP 54 (1981) 92

(16) R.W. Gianetta and H. Ikezi, Phys.Rev.Lett. 47 (1981) 849

(17) V.I. Melnikov, S.V. Meshkov, Sov.Phys. JETP 54 (1981) 505

(18) S.S. Nazin and V.B. Shikin, Sov. Phys. JETP 58 (1983) 310

(19) A.F. Andreev and A.Ya. Parshin, Sov. Phys. JETP 48 (1978) 763

(20) K.0. Keshishev, A.Ya. Parshin, and A.V. Babkin, JETP Lett. 30 (1979) 56; Sov.Phys. JETP 53 (1981) 362

(21) F. Scaramuzzi, A. Savoia, D.L. Goodstein, and M.W. Cole, Phys.Rev. B 16 (1977) 3108

(22) S. Balibar and B. Castaing, J.Phys.(Paris) Lett. 41 (1980) L 329; J.E. Avron, L.S. Balfour, C.G. Kuper, J. Landau,S.G.Lipson, and L.S. Schulman, Phys.Rev.Lett. 45 (1980) 814

(23) J. Landau, S,G. Lipson, L.M. Mäătänen, L.S. Balfour, and D.0. Edwards, Phys. Rev. Lett. 45 (1980) 31

(24) J. Bodensohn, P. Leiderer and D. Savignac, in: Proceedings of the 4th International Conference on Phonon Scattering in Condensed Matter (Springer, 1984), p. 266

(25) B. Castaing, S. Balibar, and C. Laroche, J. Physique 41 (1980) 897

(26) R. Bowley and D.0. Edwards, J.Physique 44 (1983) 723

(27) A. Andreev and V. Knizhnik, Sov.Phys. JETP 56 (1982) 2.26

(28) B. Castaing, J.Physique Lett.45(1984)L 233

(29) S. Herminghaus, diploma thesis, Univ.Mainz (1984) (unpubl ished)

(30) V.B. Shikin and P. Leiderer, Sol.St. Cominun. 47 (1983) 269

(31) H. Ikezi and P.M. Platzman, Phys.Rev. B 23 (1981) 1145, F.M. Peeters and P.M. Platzman, Phys.Rev.Lett. 50 (1983) 2021; F.M. Peeters, Phys.Rev. B 30 (1984) 159

(32) S.A. Jackson and P.M. Platzman, Phys.Rev. B 24 (1981) 499; Phys.Rev. B 25 (1982) 4886

(33) E.Y. Andrei, Phys.REv.Lett. 52 (1984)

(34) A.P. Volodin, M.S. Khaikin, and V.S. Edelman, JETP Lett. 23 (1976) 478

(35) K. Kajita, J.Phys.Soc.Jpn. 52 (1983) 372

(36) H. Etz, W. Gombert, and P. Leiderer, these proceedings. 